\title{
Beyond the Yablonovitch limit: Trapping light by frequency shift
}

\author{
Tom Markvart ${ }^{a}$ \\ School of Engineering Sciences, University of Southampton, Southampton SO17 1BJ, United Kingdom
}

(Received 20 November 2010; accepted 22 January 2011; published online 18 February 2011)

\begin{abstract}
It is shown that randomizing the photon distribution over the frequency as well as orientation variables dramatically improves the efficiency of optical confinement in a weakly absorbing material such as crystalline silicon. The enhancement in average optical path length over the Yablonovitch limit [E. Yablonovitch, J. Opt. Soc. Am. 72, 899 (1982)] is given by an inverse Boltzmann factor of the frequency shift, making it possible to manufacture, for example, efficient crystalline silicon solar cells of thickness barely $1 \mu \mathrm{m}$. (C) 2011 American Institute of Physics. [doi:10.1063/1.3554436]
\end{abstract}

Increasing the optical path lengths by surface texturing is a convenient way to increase light absorption and reduce the thickness of solar cells (see, for example, Refs. 1 and 2). Yablonovitch ${ }^{3}$ showed that the maximum path length $4 n^{2} d$ (where $n$ and $d$ are the refractive index and thickness of the layer) can be achieved by a random (Lambertian) texture. In this paper, we show that a further substantial increase in the optical path length can be achieved by photonic structure which randomizes photon momentum as well as its direction. Such scheme is based on combining a weakly absorbing semiconductor layer with a highly absorbing luminescent film and a photonic band stop mirror to trap the thermalized light. In practical terms, this allows effective light absorption close to the silicon band edge and makes it feasible to contemplate silicon-based optoelectronic devices such as solar cells which are merely $1 \mu \mathrm{m}$ thick.

Figure 1 shows a typical geometric light-trapping scheme with a textured rear surface. ${ }^{4}$ The textured surface can be pictured as scattering photons between radiation (externally coupled) and trapped (internal) photon modes, producing a uniform distribution of photons over all directions. When incident from within onto the top surface, photons in modes with directions outside the "escape cone" are reflected while photons within the cone are emitted from the structure. Neglecting reflection of rays inside the escape cone, one obtains an average reflection coefficient of $1 / n^{2}$. Taking into account the average photon path length $4 d$ per double passage through the layer yields the Yablonovitch result.

The light-trapping scheme described in this letter extends this philosophy by adding a frequency dimension to the geometrical coordinates. Such scheme is obtained by replacing the textured reflecting rear surface of Fig. 1 with a highly absorbing fluorescent layer of a similar refractive index as silicon which is again capped by a perfect reflector (Fig. 2). We assume that all photons incident on this layer are absorbed and all absorbed photons reemitted as fluorescence.

The isotropic emission process ensures random orientations as in the case reflection by the Lambertian surface (in other words, an equilibrium distribution of directions). The detailed balance between absorption and emission, expressed by the Kennard-Stepanov relations, ${ }^{5}$ now adds a similar element to the frequency (or energy) distribution of the photon gas within the silicon slab. Interaction between radiation and

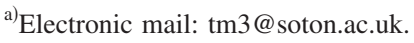

matter through repeated absorption and emission events brings the radiation into thermal equilibrium at the temperature $T_{o}$ of the absorbing/fluorescent layer (or, more precisely, its Kennard-Stepanov temperature ${ }^{5}$ ) as demonstrated, for example, in Ref. 6. Thus, the specific emission spectrum of the fluorescent layer evolves into the Bose-Einstein distribution,

$$
\rho_{\nu}=\frac{1}{e^{(\mu-h \nu) / k_{B} T_{o}}-1},
$$

where $\mu$ is the (generally nonzero) chemical potential.

The light-trapping scheme is completed by a photonic filter/mirror placed on the front face of the semiconductor slab. This mirror reflects all photons with frequency below some frequency $\nu_{o}$ but transmits all incident light with frequency above this threshold. This restriction on which photon energy is allowed to enter or escape the silicon slab parallels the angular escape cone represented by total internal reflection.

We shall now show that adding the fluorescent component dramatically extends the path length $\ell$ of photons in the structure. Let us suppose that light absorption in the semiconductor is weak $(\alpha \ll 1 / d$, where $\alpha$ is the absorption coefficient). The probability of photon absorption rather than escape through the front face is then equal to

$$
p=\frac{\dot{N}_{a b s}}{\dot{N}_{a b s}+\dot{N}_{e m}},
$$

where $\dot{N}_{a b s}$ is the rate of absorption and $\dot{N}_{e m}$ is the rate of photon emission by the structure. Clearly,

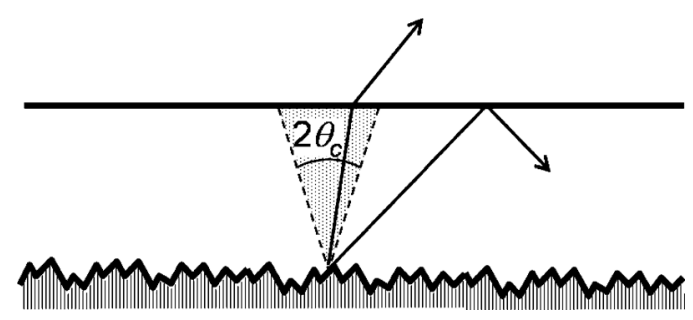

FIG. 1. A schematic depiction of a light-trapping scheme with back surface texturing and reflector $\left(\sin \theta_{c}=1 / n\right)$. 


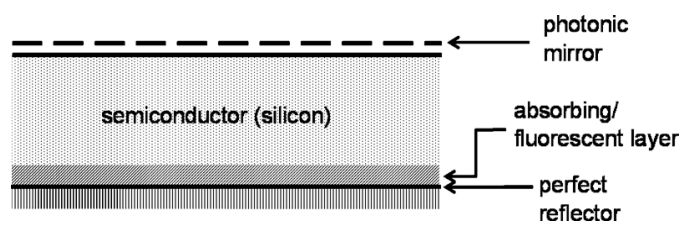

FIG. 2. The light-trapping scheme with a frequency shift.

$$
\dot{N}_{e m}=\pi A \int_{\nu_{o}}^{\infty} \frac{2 \nu^{2}}{c^{2}} \rho_{\nu} d \nu \cong \frac{2 \pi A \nu_{o}^{2} k_{B} T_{o}}{h c^{2}} e^{\left(\mu-h \nu_{o}\right) / k_{B} T_{o}}
$$

and

$$
\begin{aligned}
\dot{N}_{a b s} & =V \int_{\nu_{g}}^{\infty} \frac{8 \pi n^{2} \nu^{2}}{c^{2}} \alpha_{\nu} \rho_{\nu} d \nu \\
& \cong V \frac{8 \pi n^{2} \nu_{g}^{2} k_{B} T_{o}}{h c^{2}} \alpha\left(T_{o}\right) e^{\left(\mu-h \nu_{g}\right) / k_{B} T_{o}},
\end{aligned}
$$

where

$$
\alpha\left(T_{o}\right)=\int_{\nu_{g}}^{\infty} \alpha_{\nu}\left(\frac{\nu}{\nu_{g}}\right)^{2} e^{h\left(\nu_{g}-\nu\right) / k_{B} T_{o}} d\left(\frac{h \nu}{k_{B} T_{o}}\right)
$$

is the thermally averaged absorption coefficient, $\nu_{g}=E_{g} / h$ is a frequency corresponding to the bandgap $E_{g}$ of the semiconductor, $A$ is the area of the front face, and $V$ is the volume of the structure. The second, approximate, equalities in Eqs. (3) and (4) hold for weak to moderate light intensities when stimulated emission can be neglected.

We now note that absorption probability (2) can be written as

$$
p=\frac{\alpha\left(T_{o}\right) \ell}{1+\alpha\left(T_{o}\right) \ell} .
$$

Substituting Eqs. (3) and (4) into Eq. (2) then gives

$$
\ell=4 n^{2} d\left(\frac{\nu_{g}}{\nu_{o}}\right)^{2} e^{h \Delta \nu / k_{B} T_{o}},
$$

where $\Delta \nu=\nu_{o}-\nu_{g}$. It is seen that neither $p$ [Eq. (6)] nor $\ell$ [Eq. (7)] depends on the chemical potential $\mu$. Equation (7) represents the principal result of this work. It shows that a light-trapping scheme-consisting of a highly absorbing fluorescent layer combined with a photonic filter-extends the path length of photons in a thin semiconductor slab by a factor $\left(\nu_{g} / \nu_{o}\right)^{2} e^{h \Delta \nu / k_{B} T_{o}}$ over the corresponding Lambertian light-trapping scheme. This increase in optical path length is achieved by the confinement of photons which have been brought into thermal equilibrium at the ambient temperature.

As an illustration, Fig. 3 shows the fraction of monochromatic light at wavelength of $1 \mu \mathrm{m}$ as a function of the thickness $d$ of silicon. At this wavelength, the absorption coefficient of crystalline silicon is equal to $82 \mathrm{~cm}^{-1}$, equiva-

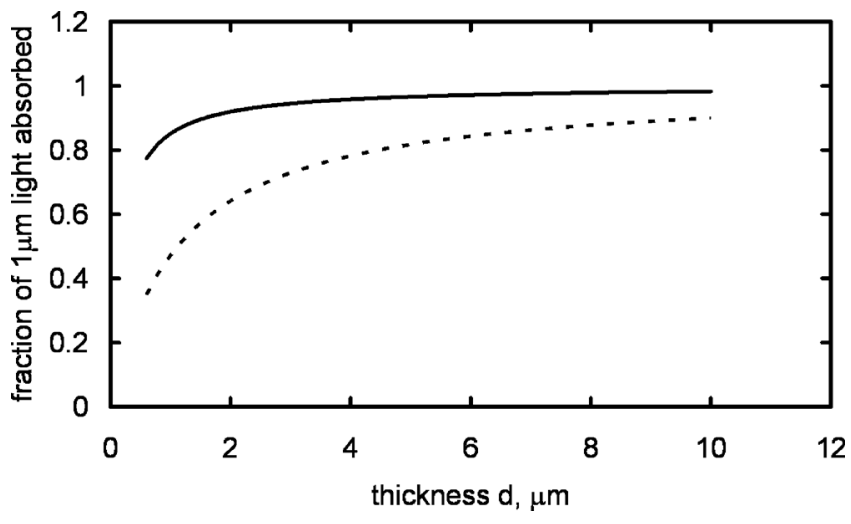

FIG. 3. The fraction of light with wavelength of $1 \mu \mathrm{m}$ as a function of the layer thickness $d$ that is absorbed in a layer with the present photonic scheme (full line) and with geometric light-trapping (dashed line).

lent to an absorption depth of $122 \mu \mathrm{m}$. To achieve $90 \%$ absorption requires a thickness of some $10 \mu \mathrm{m}$ with geometric light trapping but only $1.5 \mu \mathrm{m}$ using the present photonic scheme. Among the feasible luminescent materials, an elegant scheme for a broadband absorbing luminescent layer is realized with the use of light harvesting materials based on zeolites, ${ }^{8}$ subject to achieving a good optical coupling to silicon.

Although the photonic filter at the front aperture reflects all light with frequency below $\nu_{o}$, the effective confinement in this frequency range allows the design of ultrathin crystalline silicon solar cells with efficiencies which may equal or even surpass the theoretical limit on the traditional thick solar cells. Indeed, a photonic mirror with threshold corresponding to a wavelength close to $900 \mathrm{~nm}$ will not only ensure near-perfect absorption of light below this wavelength but also move the effective photonic bandgap closer to the ideal Shockley-Queisser maximum ${ }^{9}$ of around $31 \%$, exceeding the maximum possible efficiency of silicon solar cell by about $2 \%$. Even more importantly, it will allow material savings of more than two orders of magnitude.

The author is grateful to Dr. Marius Peters for careful reading of the manuscript.

${ }^{1}$ H. W. Deckman, C. B. Roxlo, and E. Yablonovitch, Appl. Phys. Lett. 42, 968 (1983).

${ }^{2}$ M. A. Green, High Efficiency Solar Cells (Trans Tech, Switzerland, 1987). ${ }^{3}$ E. Yablonovitch, J. Opt. Soc. Am. 72, 899 (1982).

${ }^{4}$ Light trapping by front surface texturing operates along similar lines but a rear textured surface provides a clearer demonstration of the arguments expounded in this paper.

${ }^{5}$ E. H. Kennard, Phys. Rev. 11, 29 (1918); 28, 672 (1926); B. I. Stepanov, Sov. Phys. Dokl. 2, 81 (1957).

${ }^{6}$ T. J. J. Meyer and T. Markvart, J. Appl. Phys. 105, 063110 (2009).

${ }^{7}$ See, for example, M. A. Green, Prog. Photovoltaics 10, 235 (2002).

${ }^{8}$ G. Calzaferri and K. Lutkouskaya, Photochem. Photobiol. Sci. 7, 879 (2008).

${ }^{9}$ W. Shockley and H. J. Queisser, J. Appl. Phys. 32, 510 (1961). 